\title{
Diffusione della dialisi peritoneale in Italia e nel mondo, suoi limiti e loro cause
}

\author{
Giancarlo Ruggieri
}

Nefrologo, Roma

\begin{abstract}
SPREAD OF PERITONEAL DIALYSIS IN ITALY AND IN THE WORLD, LIMITATIONS AND CAUSES
ABstract. The worldwide average use of peritoneal dialysis still accounts for $10 \%-13 \%$ of all dialysis treatments. The relationship between per capita income and PD utilization was not statistically significant; this correlation was, however, significant when PD utilization was normalized on per capita income. The reimbursements clearly play a role on dialysis choice; additionally, PD is still negatively burdened by the peritoneal pathology, due to the not-yet adequate biocompatibility of the dialysis solutions.
\end{abstract}

Key words: Peritoneal dialysis, Spread

Conflict of interest: None.

Financial support: None.

Accettato: 23 Luglio 2013

Nel 2012, Marinangeli (1) ha segnalato per il 2008, in Italia, 4094 pazienti in DP, pari al $16.7 \%$ dei prevalenti totali nei 223 centri praticanti la DP, percentuale che il Report SIN RIDT stima pari al $10.54 \%$ in tutti i Centri, ma in base a dati incompleti e con un probabile errore in eccesso. In un censimento a livello mondiale (2), nel 2008 la DP in Italia sarebbe stata del $9.6 \%$, percentuale confermata per il 2011 da un altro censimento internazionale (3). Prevalenze di DP tratte dai Censimenti ANED danno: 1984 6.3\%-1990 8.14\%; ammettendo esatto il $10.54 \%$ del 2008, l'utilizzo della DP in Italia, almeno fino al 2008, è giudicabile esiguo, ma, comunque, non lontano da quello mondiale del citato Censimento Fresenius, valutato pari all'11\%. Jain et al. (2), osservando l'andamento della DP su 130 nazioni dal 1997 al 2008, diviso in paesi in via di sviluppo e in paesi industrializzati, danno per i primi una DP $\%$ pari al $15.3 \%$ e per i secondi pari al $12.3 \%$, ed evidenziano una riduzione della DP fra il 1997 e il 2008 rispettivamente dell' $1.38 \%$ e del 5.3\%. Si potrebbe ritenere che il differente utilizzo della DP fra paesi sviluppati e paesi in via di sviluppo derivi dal differente reddito. Una personale elaborazione dei dati reperibili in Jain et al. (2) ha verificato tale ipotesi, associando alle prevalenze DP \% dei paesi il rispettivo PIL/ pro capite (PIL/pC) (3). Citandone qui i risultati fondamentali, non è emersa nessuna correlazione fra $\mathrm{PIL} / \mathrm{pC}$ e prevalenza percentuale DP, particolarmente nei paesi a più alto reddito, fra cui l'Italia; normalizzando la DP \% sul reddito secondo il metodo di Vanholder (4), è risultata, invece, una correlazione altamente significativa fra DP \% normalizzata (DPn) e PIL/ $\mathrm{pC}$, con valore DPn alto nei paesi a basso reddito e basso nei Paesi ad alto reddito: quindi, il ricorso alla DP risulta maggio- re o minore se espresso in funzione del reddito. Questo conferma che, nei paesi a maggior reddito, la scelta della DP si basa su altri criteri, essendo il reddito sufficiente per qualsiasi scelta terapeutica, la quale, quindi, dipenderà a) dal vantaggio economico dato dal rapporto rimborso/costo e b) dalla valutazione del nefrologo dell'affidabilità della DP come terapia sicura e di lunga durata. Il confronto del costo/rimborso DP vs HD appare, nel 2012, più adeguato altrove (4) che non in Italia: un decreto ministeriale del Gennaio del 2013 (5) stabilisce rapporti monetari HD/DP di cui qui si dà un esempio: HDB ospedaliera/APD 2.74 contro 1.02 in Canada e 1.316 in Francia. I rimborsi sarebbero più equi se basati sul metodo del "bundle reimbursement", adottato recentemente prima dal Portogallo e, quindi, dagli USA. In esso, una cifra forfettaria include la quasi totalità delle prestazioni necessarie, dialitiche, mediche, diagnostiche, terapeutiche farmacologiche e nutrizionali, e garantisce per legge il pieno rimborso solo con il raggiungimento di target definiti. I risultati ottenuti sono stati giudicati finora assai positivi $(6,7)$. L'utilizzo della DP è probabilmente, inoltre, condizionato dall'ancora insufficiente biocompatibilità delle soluzioni dialitiche, significativamente migliorate con l'introduzione delle nuove soluzioni e dell'icodestrina, ma, tuttora, causa di danni peritoneali e sistemici secondo un'ampia letteratura: esse sono utilizzate da venti anni, ma con un drop out non marginale (1) e un persistente rischio di EPS (8): questi problemi e i problemi economici succitati non facilitano la diffusione della DP, ma, piuttosto, favoriscono la sua stagnazione o la sua riduzione (1): più congrui rimborsi e soluzioni più biocompatibili dovrebbero indurre nuove prospettive. 


\section{Riassunto}

L'utilizzo della dialisi peritoneale nel mondo rappresenta tuttora mediamente il 10-13\% della terapia dialitica. Il rapporto fra reddito pro capite e uso della DP non dimostra una correlazione significativa, esistente se la dialisi peritoneale è normalizzata sul reddito pro capite, almeno relativamente al periodo qui considerato, 1997-2008 (2). Sembra evidente il peso del rimborso nella scelta dialitica, mentre sulla DP pesa tuttora negativamente la patologia peritoneale dovuta alla limitata biocompatibilità delle soluzioni dialitiche.

Parole chiave: Dialisi peritoneale, Diffusione
Dichiarazione di conflitto di interessi: L'Autore dichiara di non avere conflitto di interessi.

Contributi economici degli autori: L'Autore dichiara di non aver ricevuto sponsorizzazioni economiche per la preparazione dell'articolo.

Indirizzo degli Autori:

Dr. Giancarlo Ruggieri

Via degli Estensi 91

00164 Roma

giancarlo.ruggieri@mclink.net

\section{Bibliografia}

1. Marinangeli G, Cabiddu G, Neri L, Viglino G, Russo R, Teatini U. Old and new perspectives on peritoneal dialysis in Italy emerging from the Peritoneal Dialysis Study Group Census. Perit Dial Int 2012; 32: 558-65.

2. Jain AK, Blake P, Cordy P, Garg AX. Global Trends in Rates of Peritoneal Dialysis. J Am Soc Nephrol 2012; 23 (3): 533-44.

3. Fresenius Medical Care. ESRD Patients in 2011 A Global Perspective; www.vision-fmc.com/.../ESRD_Patients_in_2011.

4. Vanholder R, Davenport A, Hannedouche T, et al. Reimburse- ment of Dalyisis: a Comparison of Seven Countries. J Am Soc Nephrol 2012; 23: 1291-8.

5. Supplemento ordinario n. 8 alla Gazzetta Ufficiale, Serie generale - n. 23 - Prestazioni specialistiche ambulatoriali: 28-1-2013.

6. Ponce P, Marcelli D, Guerreiro A, et al. Converting to a capitation system for dialysis payment: the Portuguese experience. Blood Purif 2012; 34: 313-24.

7. Maddux FW. Impact of the bundled of the End-Stage Renal Disease payment system on patient care. Blood Purif 2012; 33: 107-11.

8. Garosi G. SIN GDS Dialisi Peritoneale. Peritonite Sclerosante Incapsulante. Nephromeet 2013-04-21. 